\title{
34. Studies on Reciprocal and Various Inhibiting Influences on the Growth of Tumors Exerted by Inoculation of Various Transplantable Tumors.
}

\author{
By Ryōsuke Yokota. \\ (Comm. by T. SASAKI, M. I. A., Nov. 12, 1947.)
}

\section{Résmé}

Using several kinds of transplantable tumors of the rat family, I made researches into the influence on the growth of later-inoculated tumors exerted by absorption of previously inoculated tumors, considering how the relation between primary and later inoculation was influenced by the quantities and periods of the inoculations. I obtained the following results :

Those tumors with originated from more closely related organs and species caused more intensive phenomena of Inhibiting influence.

Experiment I. Relations between the same kind of sarcoma of the same genus.)

The 1st series of experiments was carried out on the white mouse, with the white-mouse-sugar-sarcoma ${ }^{12}{ }^{2)}$ ) (Fig. A) as material. Observations extended to the effect of inoculations made as late as the 124 th day from the 1st inoculation, but not the slightest growth of the reinoculated sarcoma of the same kind could be found, even when the quantity inoculated first was very small. (Fig. I.-IV.)

Experiment II. (Relations between sarcoma and carcinoma of the same genus)

At a time when the inhibiting influence on the growth of the white-mouse-sugar-sarcoma was, according to my judgement, certainly still efficient, the mice were inoculated with the white-mouse-Bashford-carcinoma $^{4}$ (Fig. B; breast carcinoma). In these cases the influence was found to be very slight. (Fig. III.-V.)

Experiment III. (Relations between different kinds of sarcomas of the rat and the mouse.)

Inhibiting influence on the growth of tumors was found to be exerted reciprocally by two sarcomas of different species viz. the white-mouse-sugar-sarcoma and the white-rat-Fujinawa-sarcoma ${ }^{5)}$ (Fig. C). But it was very much weaker than the influence of the same kind of sarcoma, and it became imperceptible after a definite term. (Fig. VI.-IX.)

1) Nishiyama, Y.: Experimentelle Erzeugung des Sarcoms bei Ratten durch wiederholte Injection von Glucoselosung. Gann 32, 85: 1938.

2) Takizawa, N.: Uber die Erzeugung des Sarcoms der Maus und Ratten durch wiederholte subcutane Injection der konzentrierten Zucker]osung. Gann 32, 236: 1938.

3) Nagayo, M.: On Heteroplastic Transplantation of Mouse Sarcoma. Gann 35, 232 : 1941. 35, 295 : 1941.

4) Bashford, E.F., J. A. Murrey und M. Healand: Ergebnisse der Experimentelle Krebsforschung. Z. f. Immunitatf. 1, 449 : 1909. ioc. cit.

5) Fujinawa, K.: Beitrag، zur kenntnis der Transplantation und Wucherung des Rattensarcoms. Kyoto-igaku-Zasshi 16, 1220 : 1919. 
Experiment IV. (Relations between breast carcinomas of mice of two different strains.)

The white-mouse-Bashford-breast carcinoma was inoculated into white mice which previously had been inoculated with the breast carcinoma $^{1)}$ of the d.b.r. strain-brownish-gray-mouse (Fig. D) and had absorbed it. In these cases the intensity of the inhibiting influence was rather inferior to that found in experiment III. (Fig. X.)

Exp. Relation between Pr.

No. \& La. inoculation Intensity of inhibiting influence

I. The same kind of Most intensive inhibiting influence: Not even the slightest $\mathrm{Sa}$. growth of the reinoculated tumors was to be found.

II. Sa. and $\mathrm{Ca}$. of the Very slight inhibiting influence: Developments of tumors same genus were somewhat inferior to, yet not very different from those of the contrasting experiments :

III. Different kinds of Influence less intensive than that found in Experiment I. and Sa. of the rat and becoming imperceptible after a definite term: Some percents the mouse of tumors showed positive transplantability, but their growth was comparatively small. In case of inoculation after a definite term no inhibiting influence was to be found.

IV. Breast Ca. of mice Inhibiting influence less intensive than that found in Experiof two different ment III.: The transplantability of tumors remained unimstrains paired in most cases, but their growth was comparatively small.

1) Kinoshita, R.: Advance of medicine. (1) 1943. loc. cit. 


\section{Experiment I.}

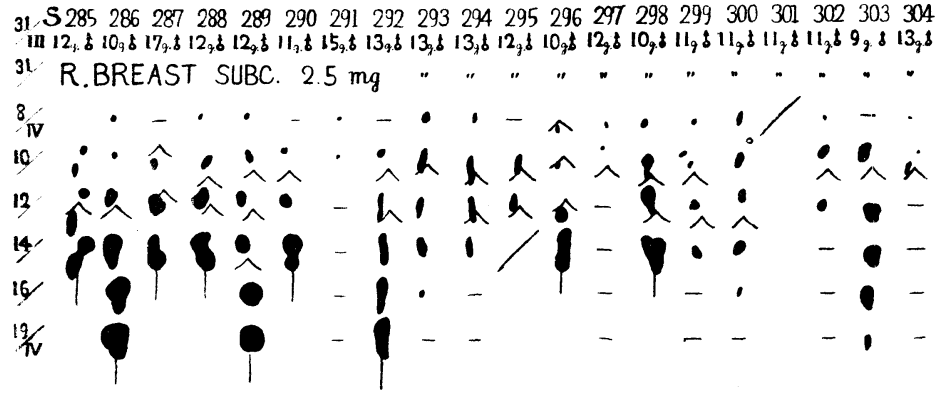

$15, \mathbf{S} 291293294299300302 \quad 303 \quad 304$
$18,816,815,8 \quad 16,812,8 \quad 13,8 \quad 14,812,8$

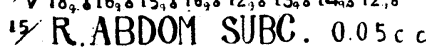

25. - $-\ldots \ldots$

27 - $-\ldots+\ldots$

$3 / v-$

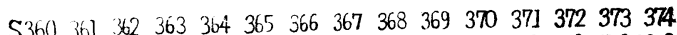
$17,915,915,9 \quad 17,917,917,919,920,915,915,919,919,920,915,916,9$

R. ABDOM. SUBG. 0.05 c.c. "

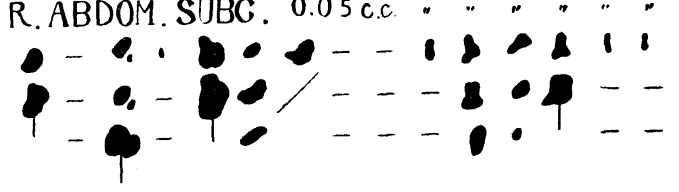

Fig. I. The inoculation of the mouse-sugar-sarcoa (S285-S304). Its reinoculation (S291-S304) and its contract (S360-S374).

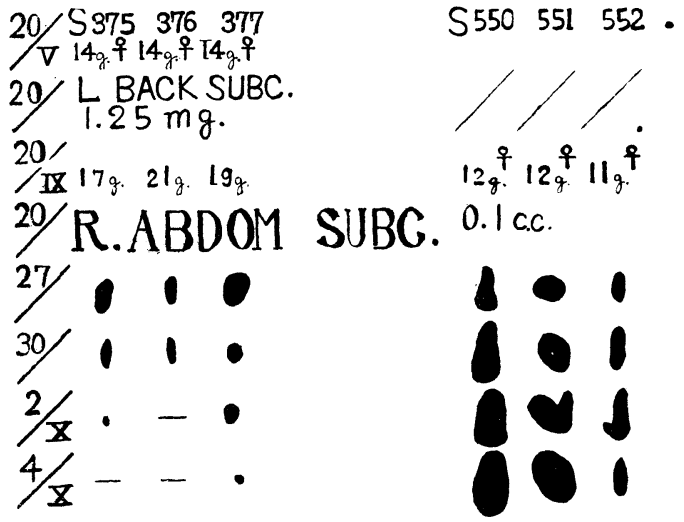

Fig. II. The reinoculation (S375-S377) and the contrast-inoculation (S550-S552) with the mouse-sugar-sarcoma.

(Ehe original figures of tumors were sketched by palpation methyds). 
Experiment II.
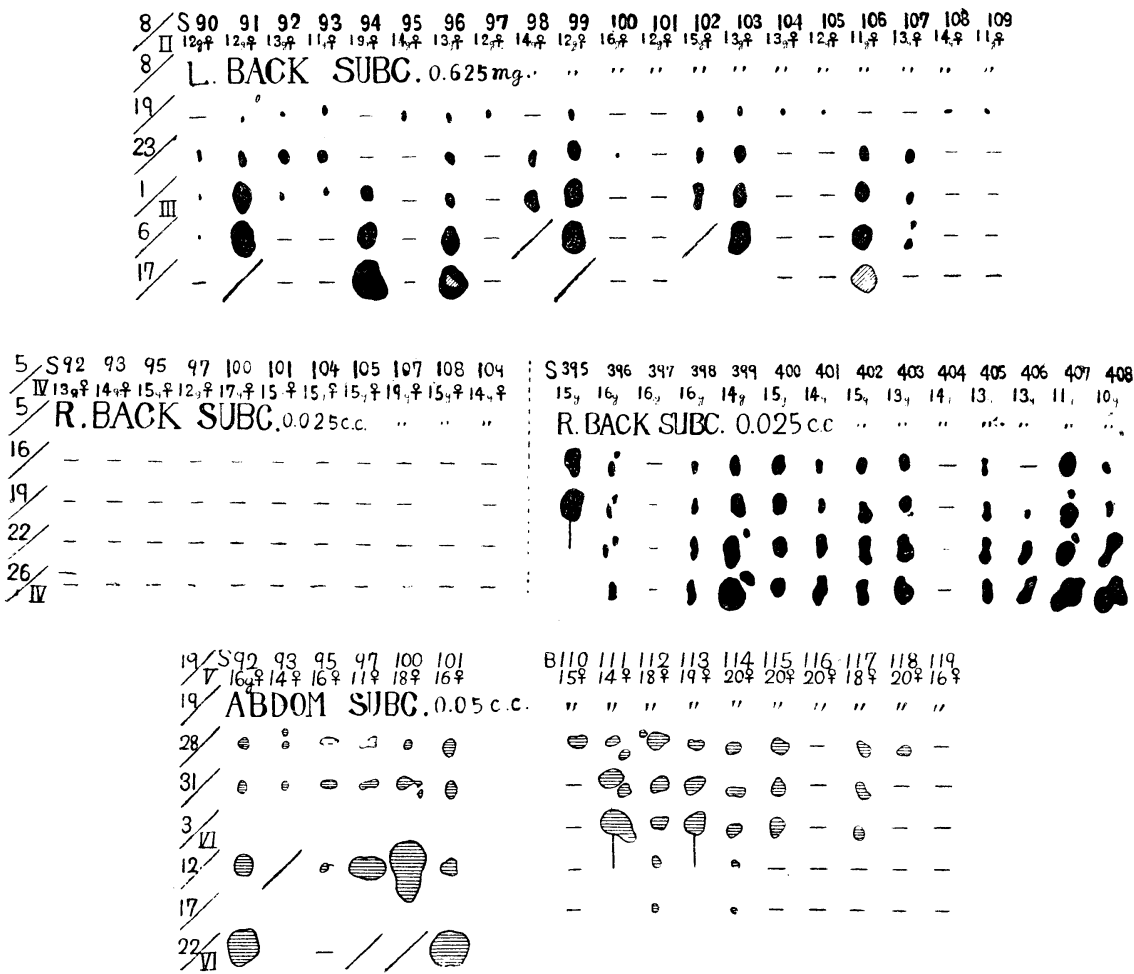

Fig. III. The inoculation of the mouse-sugar-sarcoma (S90S109). Its reinoculation (S92-S102) and its contrast (S395-S408). The inyculation of the Bashfordcarcinoma into the mice (S92-S101) and into the contrast-mice (B110-B119).
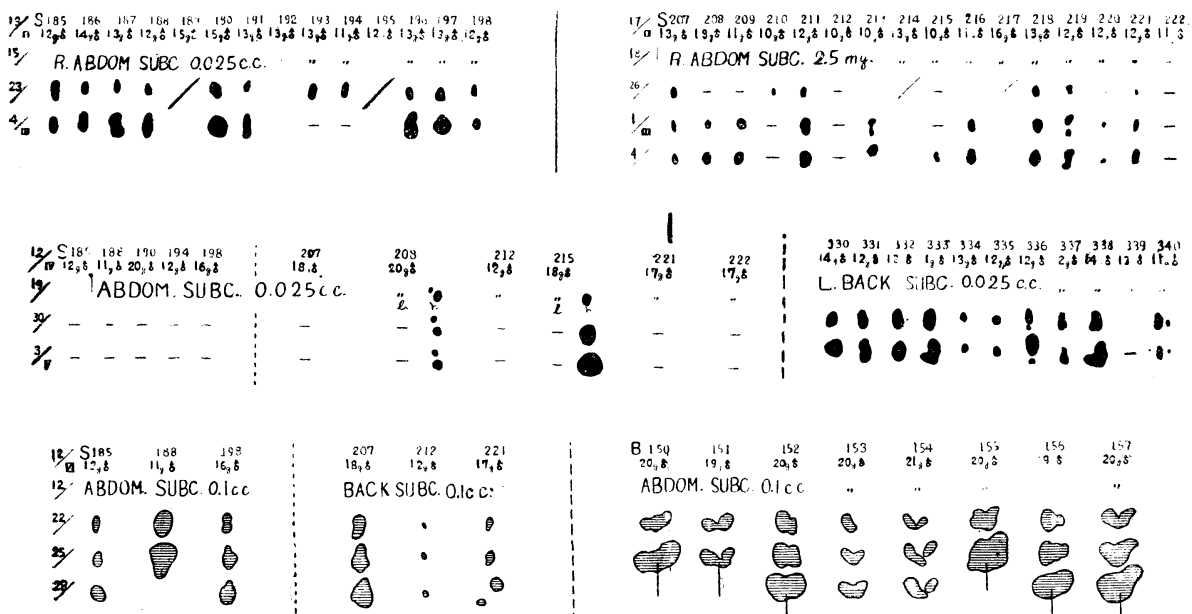

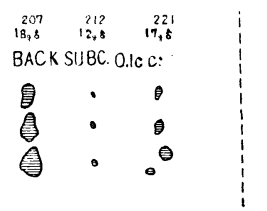

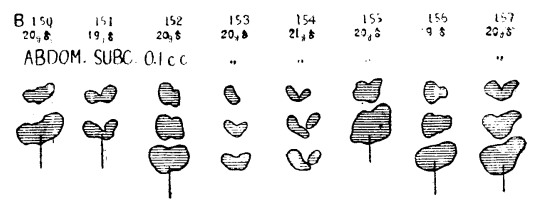

Fig. IV. The inculation of the mouse-sugar-sarcoma (S185-S193, S207-S222). Its reinoculation (S185-S198, S207-S222) and its contrast $(\mathrm{S} 330 \backsim \mathrm{S} 340)$. The inoculation of the Bashford-carcinoma into the mice (S185-S193, S207-S221) and into the contrast-mice (B150-B157). 


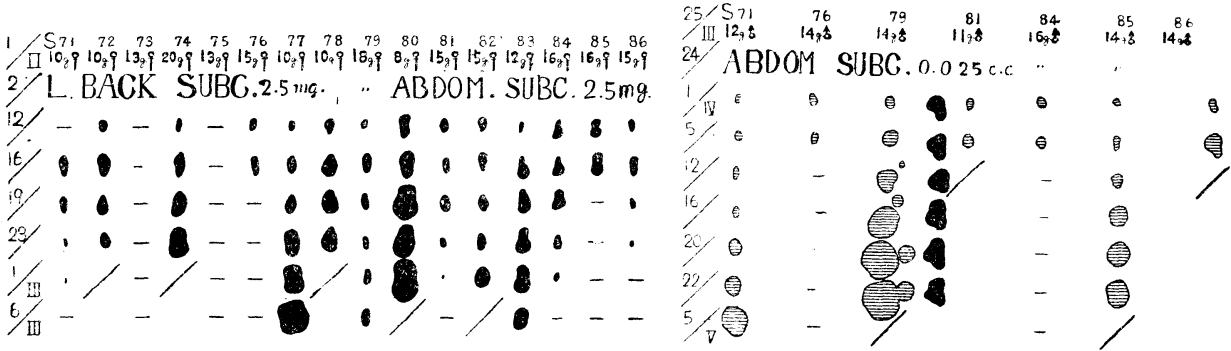

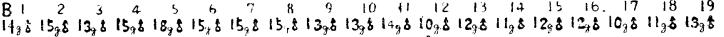

$$
\begin{aligned}
& \text { BACK SUBC. } 0.025 c \text { ". "ABDOM SUBC. } 0.025 \text { c.c." " }
\end{aligned}
$$

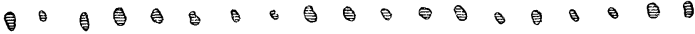

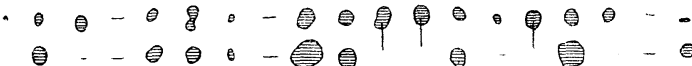

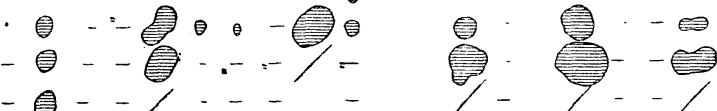

Fig. V. The inoculation of the mouse-sugar-sarcoma (S71-S86). The inoculation of the Bashford-carcinoma into the mice (S71-S86) and contrast-mice (B1-B19).

\section{Experiment III.}

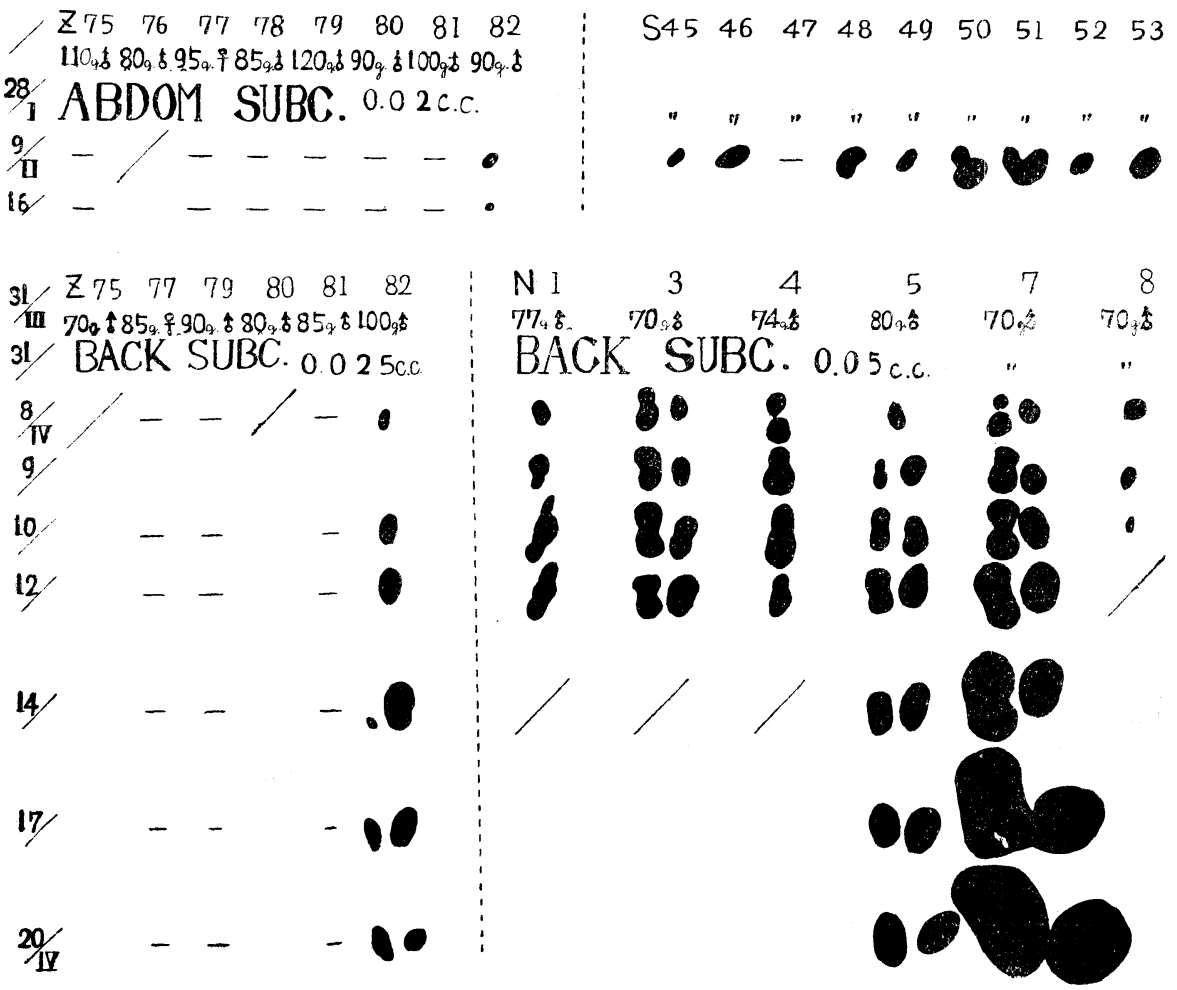

Fig. VI. The inoculation of the mouse-sugar-sarcoma into the rats (Zgroup) and into the mice (N-group). The inoculation of the rat-Fujinawasarcoma into the rats (Z-group) and into the contrast-rats ( $N$-group). 

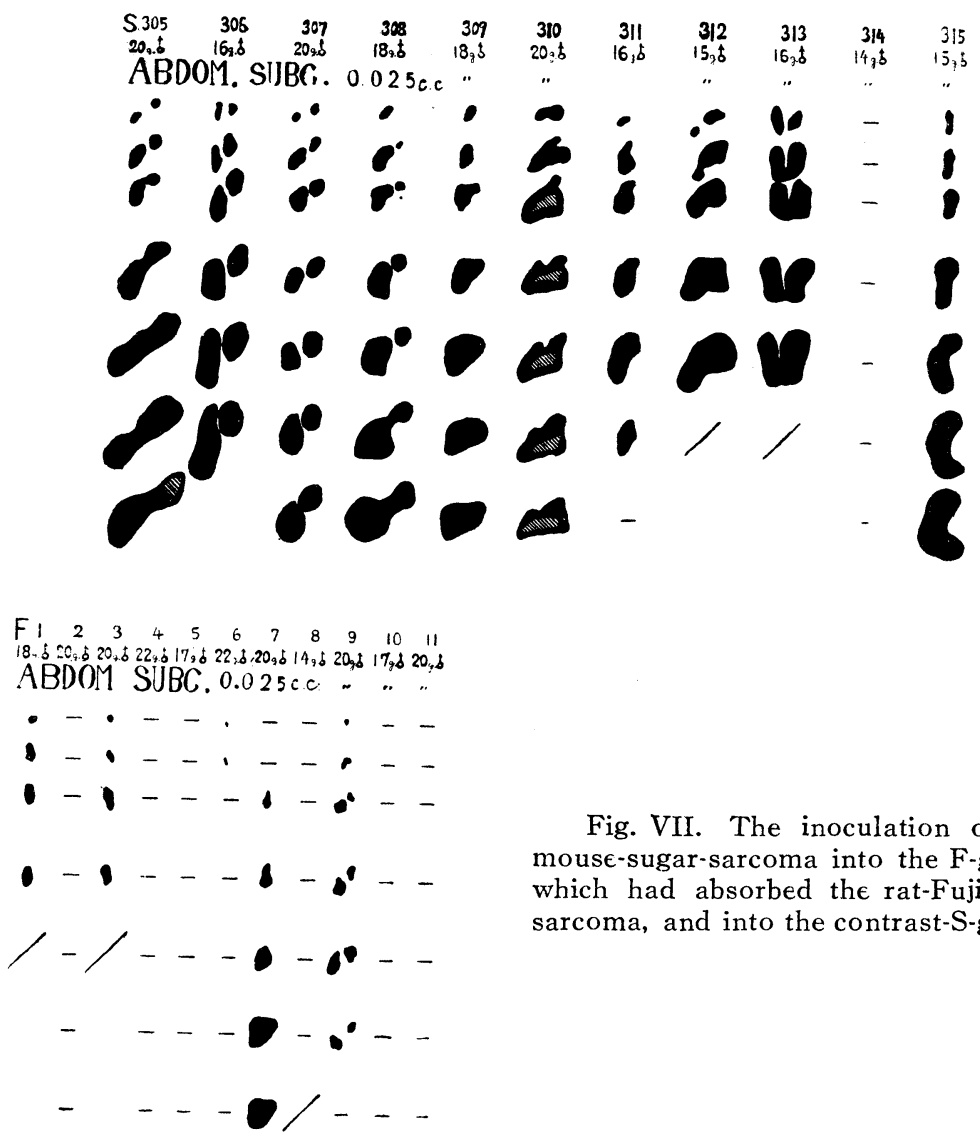

Fig. VII. The inoculation of the mouse-sugar-sarcoma into the F-group, which had absorbed the rat-Fujinawasarcoma, and into the contrast-S-group.

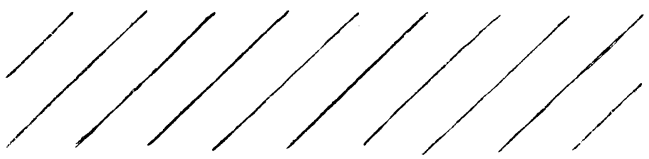

R.BACK SUBC. $125 \mathrm{mg}$.

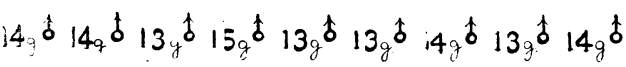
L.BACK SUBC. $2.5 \mathrm{mg}$. R. ABDOM SUBC. 0.05 c.c." "

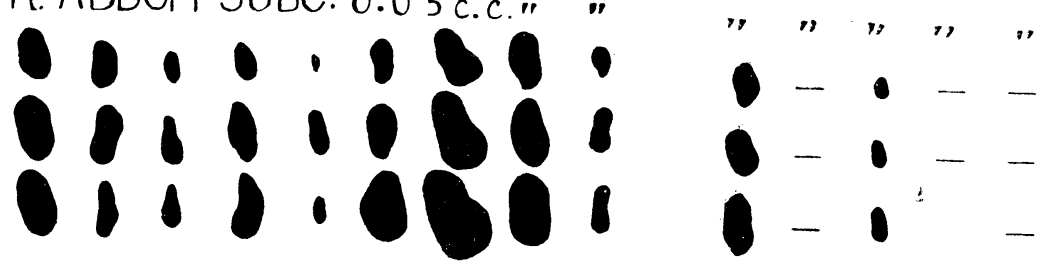

Fig. VIII. The inoculation of the mouse-sugar-sarcoma into the F-group, which had twice absorbed the rat-Fujinawa-sarcoma, and into the contrast-S-group. 


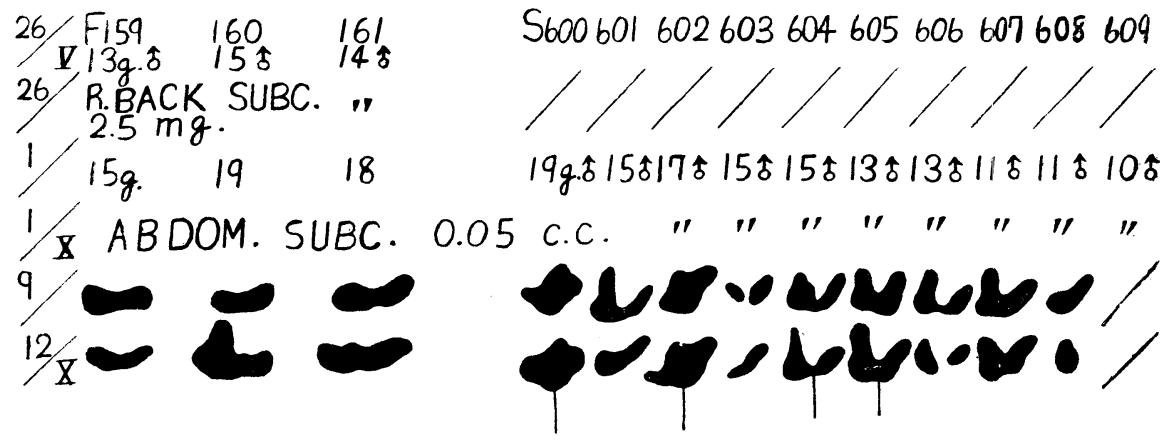

Fig. IX. The inoculation of the mouse-sugar-sarcoma into the F-group, which had absorbed the rat-Fujinawa-sarcoma, and into the contrast-S-group.

Experiment IV.
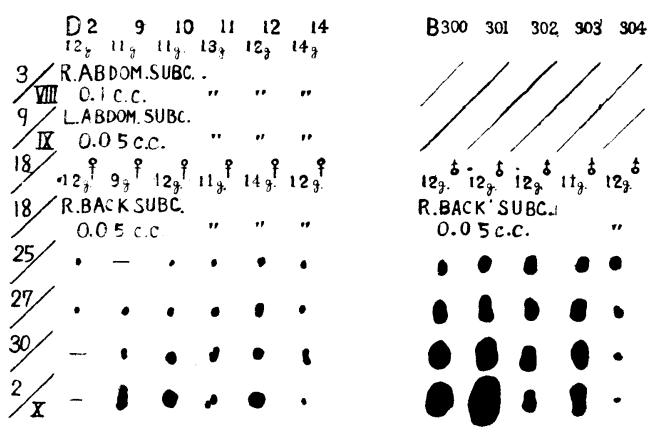

$\begin{array}{llllllllll}305 & 306 & 307 & 308 & 309 & 310 & 311 & 312 & 313 & 314\end{array}$

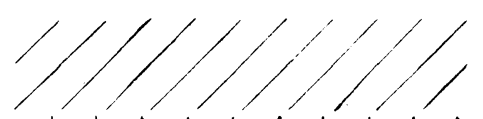

$12 g^{b} 12 g^{b} 12 g^{b} 11 g^{b} 11 g^{b} 12 g^{b} 11 g^{b} 12 g^{t} 13 g^{\delta} 11 g^{b}$

L.ABDOM.SUBC.

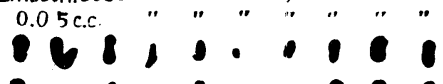

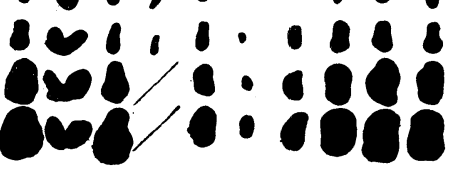

Fig. X. The inoculation of the white-mouse-breast-carcinoma into the D-group, which had twice absorbed the d.b.r. mouse-breastcarcinoma, and into the contrast-S-group. 


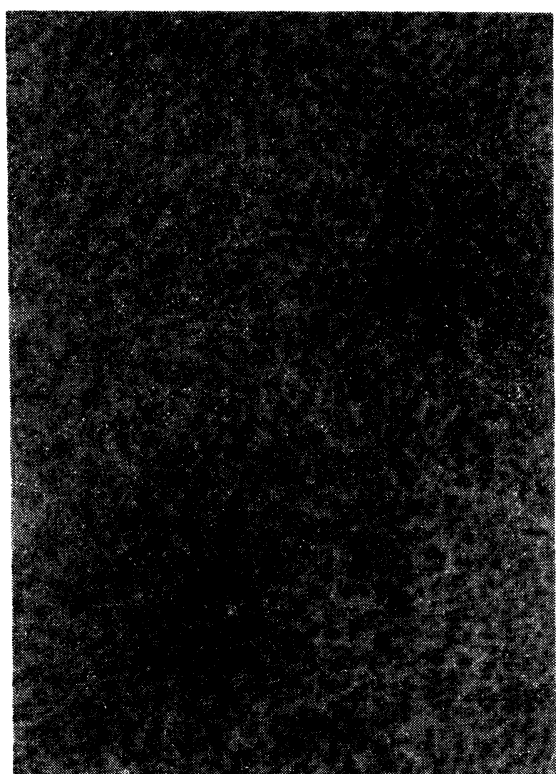

$8: 10$

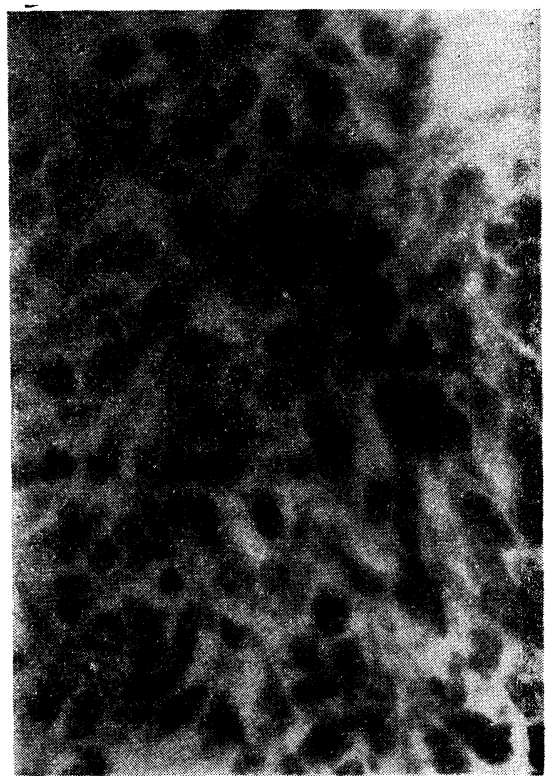

$40 \times 10$

Fig. A : Sugar-sarcoma.

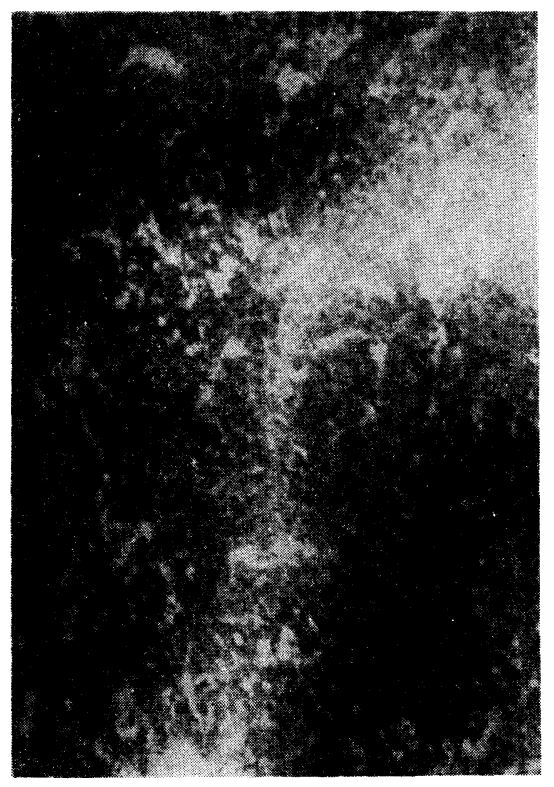

$8 \times 10$

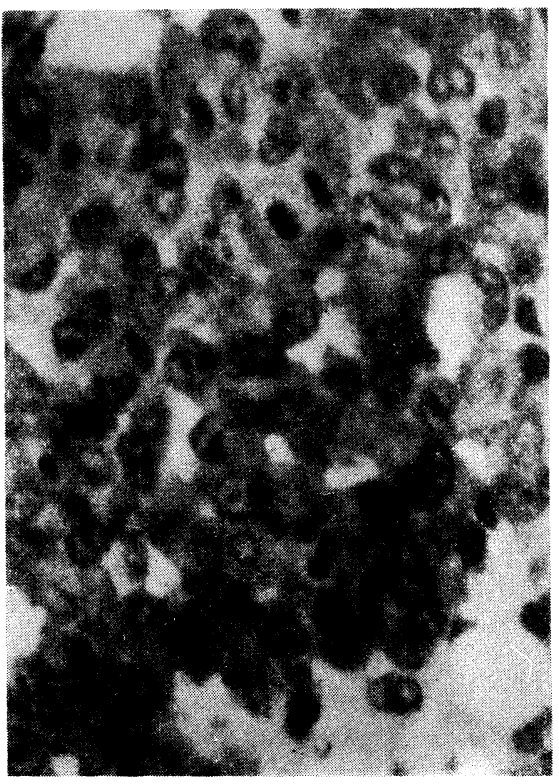

$40 \times 10$

Fig. B : Bashford-carcinoma. 


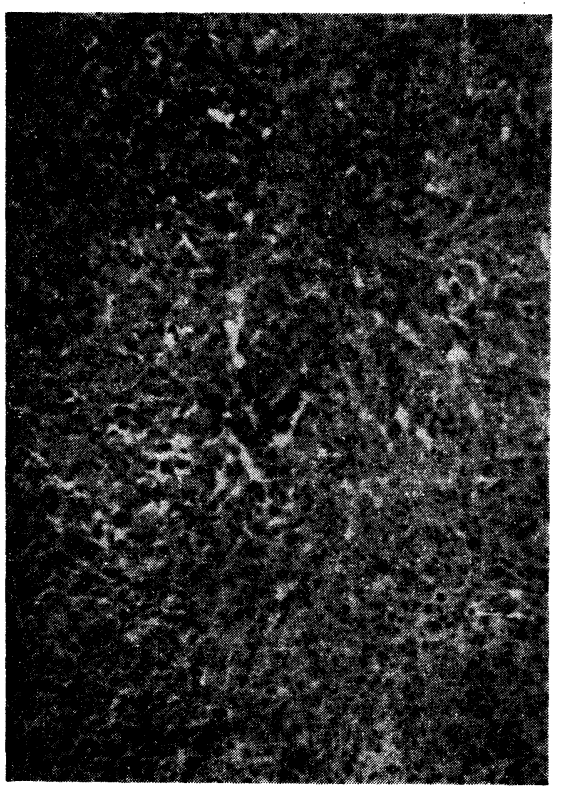

$8 \times 10$

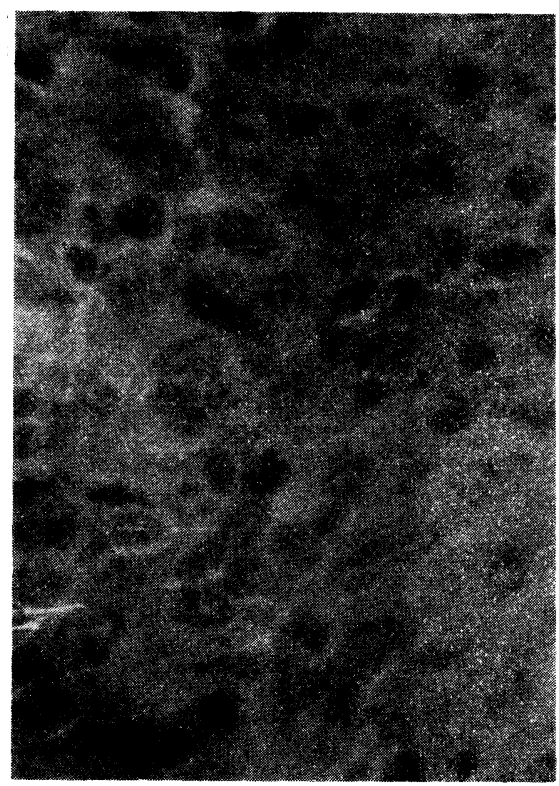

$40 \times 10$

Fig. C : Fujinawa-sarcoma.

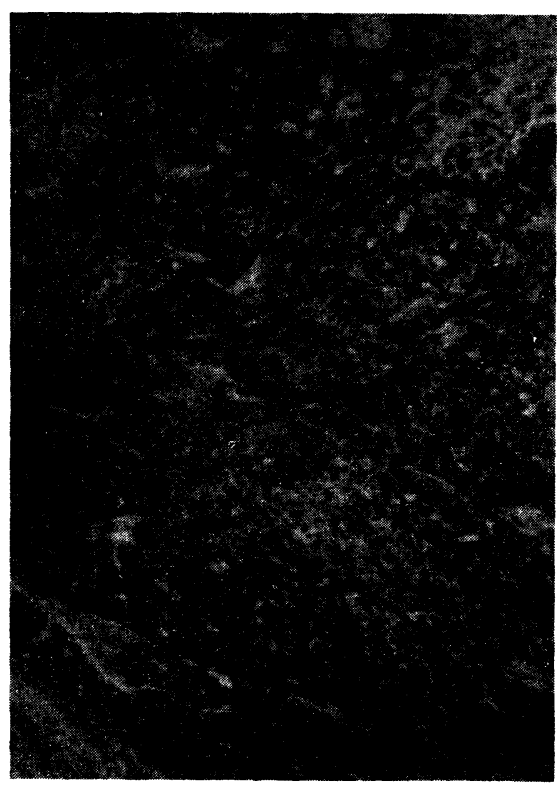

$8 \times 10$

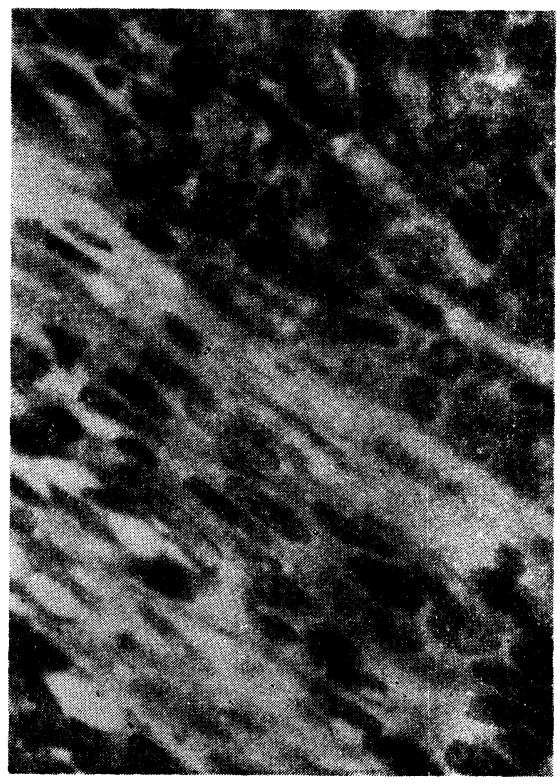

$40 \times 10$

Fig. D : d.b.r.-carciroma 